\title{
Successful Treatment of Nail Lichen Planus with Alitretinoin: Report of 2 Cases and Review of the Literature
}

\author{
Adel Alsenaid Irina Eder Thomas Ruzicka Markus Braun-Falco Ronald Wolf \\ Department of Dermatology and Allergology, Ludwig Maximilian University Munich, Munich, Germany
}

Key Words

Alitretinoin · Lichen planus · Nails

\begin{abstract}
Background: Treatment of nail lichen planus (LP) is difficult and an optimal therapy is lacking. Objective: To report additional cases to the scant existing literature to learn more about therapeutic options for nail LP. Methods: A regimen of $30 \mathrm{mg}$ alitretinoin daily in 2 cases of nail LP over a period of 9 and 8 months, respectively. Results: In either case, nail changes showed marked improvement under oral alitretinoin therapy within 2 and 4 months, respectively. In both patients, affected nails with end-stage destructive pterygium were resistant to any previously applied therapy. Conclusion: Alitretinoin is an effective treatment option for nail LP. We recommend early diagnosis of nail LP and early initiation of systemic therapy with alitretinoin to prevent the development of pterygium and permanent nail damage. However, further clinical studies are needed to establish reliable guidelines for nail LP therapy.
\end{abstract}

(c) 2014 S. Karger AG, Basel

\section{Introduction}

Lichen planus (LP) is a common chronic inflammatory mucocutaneous condition of unknown origin that usually affects mid- dle-aged patients. LP was first described by Erasmus Wilson in 1869; it presents with a variety of clinical manifestations affecting the skin, mucous membranes, nails and hair [1]. Nail involvement occurs in $10 \%$ of patients, in approximately $4 \%$ with permanent damage to at least one nail. The nails may also be affected in the absence of cutaneous symptoms such as destructive inflammatory onychodystrophy [2, 3]. LP of the nail affects the quality of life considerably. Treatment of nail LP is difficult and an optimal therapy is lacking. We report 2 cases of nail LP that were successfully treated with alitretinoin. Using PubMed, a review of the English literature revealed one case report of nail LP treated with alitretinoin [4] and another case of oral, esophageal and cutaneous LP controlled with alitretinoin that also showed nail improvement [5]. Our objective is to report additional cases to the scant existing literature to learn more about therapeutic options for nail LP.

\section{Case Reports}

Case 1

A man in his 50 s reported a 30 -month history of mucous membrane and nail changes. His past medical history was noncontributory and he was not on any regular systemic medication. Clinical examination showed an involvement of all finger- nails and toenails with onychodystrophy, discoloration, longitudinal ridging, striations, splitting and thinning, some nails also exhibited pterygium (fig. 1a). Mycological examination of the nail was negative. The diagnosis of LP with oral and nail involvement was made based on these clinical features. Oral lesions were treated with topical corticosteroids and showed good clinical response. Nail involvement showed resistance to the topical application of high-potency corticosteroids and tacrolimus $0.1 \%$ ointment. After 5 months, oral alitretinoin $30 \mathrm{mg} /$ day was initiated, which led to a significant improvement of most nails. The nail changes began to resolve within 2 months after alitretinoin administration. Alitretinoin therapy continued over a total period of 9 months and was well tolerated with no side effects. A significant improvement of most nails was observed after 5 months of therapy (fig. 1b). Affected nails with pterygium showed resistance to therapy. Follow-up over 1 year revealed a stable nail condition under topical mometasone solution to maintain the effect of alitretinoin therapy.

\section{Case 2}

A woman in her 60s with a 27-year history of LP presented with typical lesions on the skin, oral cavity and nails. Except for multiple allergies (type I hypersensitivity) and subclinical autoimmune hypothyroid-

\section{KARGER}

E-Mail karger@karger.com

www.karger.com/drm
(C) 2014 S. Karger AG, Base

1018-8665/14/2294-0293\$39.50/0
Adel Alsenaid

Department of Dermatology and Allergology

Ludwig Maximilian University Munich, Frauenlobstrasse 9-11

DE-80337 Munich (Germany)

E-Mail dr.senaid@gmail.com 
Fig. 1. Two cases of nail LP managed with alitretinoin: prior to oral administration of alitretinoin with resistance to topical treatment $(\mathbf{a}, \mathbf{c})$ and after treatment with alitretinoin $30 \mathrm{mg}(\mathbf{b}, \mathbf{d})$.

Fig. 2. Histology from a skin biopsy of the second case demonstrates classic features of LP such as orthohyperkeratosis, wedgeshape hypergranulosis, acanthosis with a sawtooth rete ridge pattern, band-like lymphoid infiltrate with interface destruction and the presence of Civatte bodies (hematoxylin-eosin, $\times 100$ ).

ism, her past medical history was noncontributory. Clinical examination showed involvement of all fingernails and toenails with severe onycholysis and onychodystrophy, chromonychia with yellow-brown discoloration, longitudinal ridging, striations, splitting and thinning, some nails showing severe pterygium (fig. 1c). There
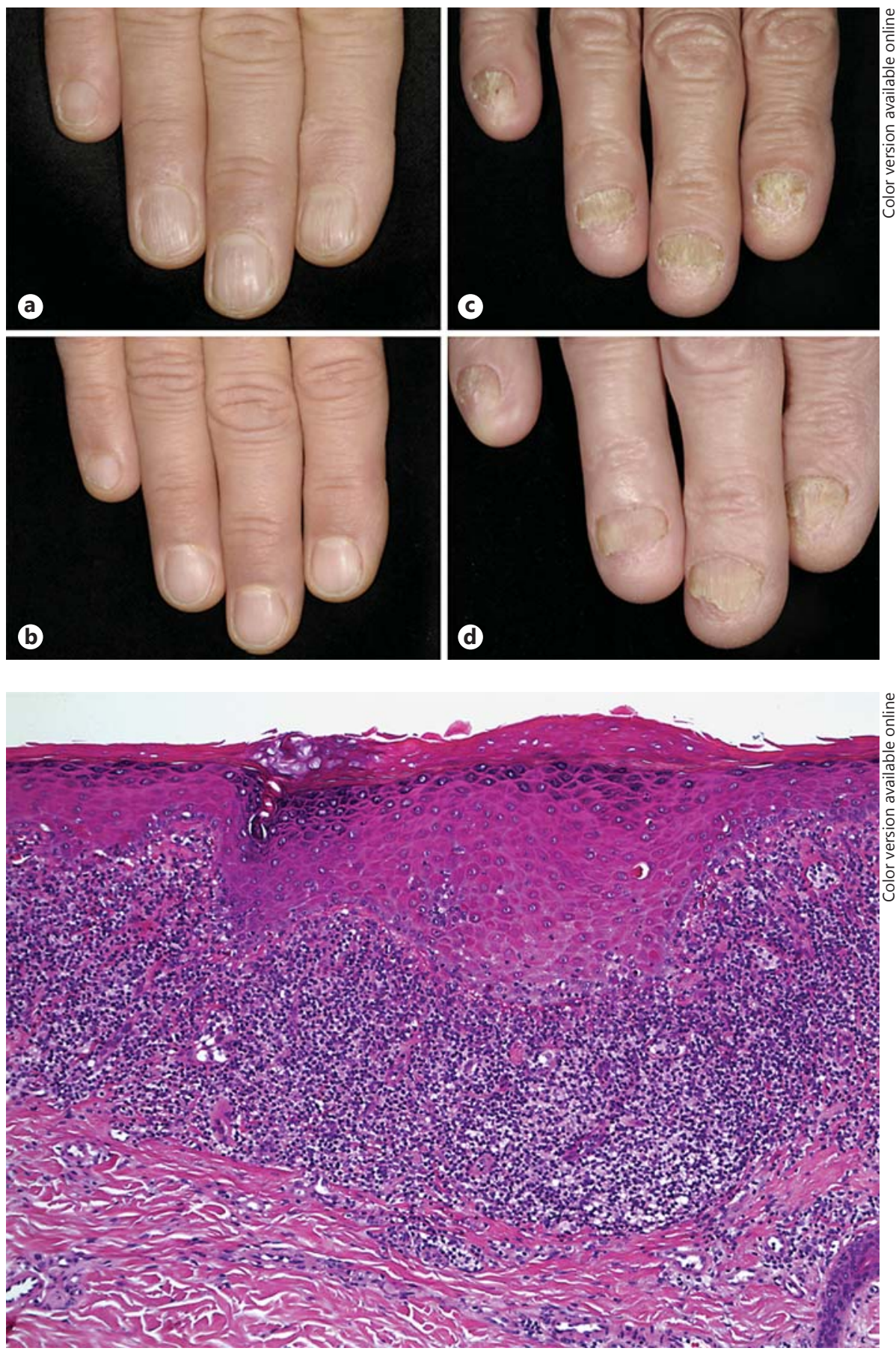

were also typical skin changes on the patient's arms with mucous membrane involvement. The result of a skin biopsy was consistent with LP (fig. 2). Mycological examination of the nails was negative. The diagnosis of nail LP was made based on clinical appearance and skin histology. Treatment with topical applications of corticosteroids resulted in a significant improvement of the skin and oral lesions but without any effect on the nails. Tacrolimus $0.1 \%$ ointment was also applied to the nails without any clinical improvement. Treatment with oral alitretinoin $10 \mathrm{mg} /$ day was started, which was increased to $30 \mathrm{mg}$ /day after 3 months. Treatment was continued 
for a total of 8 months. The nail changes improved after alitretinoin administration within 4 months (fig. 1d), and the patient's thyroid hormone levels remained normal. However, since the patient complained of headache, elevated liver enzymes and abdominal pain that later developed into acute diverticulitis, treatment with alitretinoin was discontinued, which led to worsening of her cutaneous LP and was managed by topical corticosteroids. Her nail LP was stable although pterygium remained resistant to therapy. The patient refused to adhere to long-term follow-up.

\section{Discussion}

Here we report on 2 cases of nail LP involving all nails that were successfully treated with oral alitretinoin, adding them to one previous case report by Pinter et al. [4]. The typical clinical features in each case and histologic features of a skin biopsy in the second case were diagnostic of nail LP. Nail LP affects the quality of life considerably due to impaired manual activity, cosmetic discomfort, the chronic course of the disease and numerous recurrences. Different treatment modalities have been used in nail LP: although they are usually ineffective, topical corticosteroids are commonly considered as firstline treatment for nail LP. For involvement of up to three nails, intralesional steroid injections can be considered, but are very painful and relapses are common [3]. If more than three nails are involved, systemic corticosteroids are considered as first-line treatment; however, prolonged or repeated use may cause considerable side effects $[6,7]$. There is a single case re- port of using tacrolimus ointment successfully in nail LP [8]. Another report of a patient with refractory nail LP showed significant improvement after etanercept monotherapy [9], although some studies described etanercept as a trigger factor for LP [10]. In 2 patients, LP involving the nails improved with methotrexate (10-20 mg subcutaneously once weekly) a few weeks after initiation [11]. Etretinate and antimalarials were also reported $[12,13]$. Although acitretin is a known treatment modality for LP, an effect on nail LP is lacking [14]. No curative therapy is known and the treatment of nail LP is challenging due to the lack of treatment guidelines. Frequent relapses are common. Autoimmune hypothyroidism may contribute to the development of LP with a possible underlying associated mechanism [15]. Information about the efficacy of systemic treatments in nail LP is scarce because most studies on LP did not focus on nail changes. Alitretinoin (9-cis-retinoic acid) is an endogenous vitamin A derivative which regulates cell proliferation and differentiation. Its anti-inflammatory and immune-modulating effects result from the control of pro-inflammatory cytokine production by keratinocytes and modulation of leukocyte activity. Therefore, it has been approved for the oral treatment of moderate to severe chronic hand eczema that is unresponsive to potent topical corticosteroids [16]. The benefit of systemic retinoids for LP is supposed to result from their antiproliferative mode of action mediated by binding to nuclear receptors (RAR and RXR) and there is some evidence of receptor expression in the nail matrix [4]. The positive effect of alitretinoin on skin, oral, esophageal and nail LP has already been described [5]. In our 2 cases, the patients showed nail LP which was refractory to classic treatment options (topical corticosteroid and tacrolimus ointment $0.1 \%$ ). We therefore chose a regimen of $30 \mathrm{mg}$ alitretinoin daily in both cases over a period of 9 and 8 months, respectively. In the second case, the patient did not tolerate systemic alitretinoin $30 \mathrm{mg}$ daily and developed systemic side effects as well as acute abdominal symptoms which were most likely unrelated to alitretinoin. However, dose adjustment would be an option after stabilizing her acute symptoms. The patient was not compliant enough for long-term followup. In either case, nail changes showed marked improvement under oral alitretinoin therapy within 2 and 4 months, respectively. In both patients, affected nails with end-stage destructive pterygium were resistant to any applied therapy. Together, the significant clinical improvement in our 2 cases suggests that alitretinoin is an effective treatment option for nail LP. We recommend early diagnosis of nail LP and early initiation of systemic therapy with alitretinoin to prevent the development of pterygium and permanent nail damage. However, further clinical studies are needed to establish reliable guidelines for nail LP therapy as well as an improved scoring system for nail LP to assess treatment efficacy.

\section{Disclosure Statement}

T.R. has acted as a principal investigator, speaker and consultant for Basilea Pharmaceutica.

\section{References}

1 Wolf R, Ruzicka T, Rupec RA: Pleomorphismus des Lichen ruber - klinische Variationsbreite, Pathogenese und Therapie [The chameleon's many faces - clinical spectrum, pathogenesis and therapy of lichen planus]. Aktuelle Derm 2010;36:180-185.

2 Goettmann S, Yaraa I, Moulonguet I: Nail lichen planus: epidemiological, clinical, pathological, therapeutic and prognosis study of 67 cases. J Eur Acad Dermatol Venereol 2012;26: 1304-1309.
3 Piraccini BM, Saccani E, Starace M, Balestri R, Tosti A: Nail lichen planus: response to treatment and long term follow-up. Eur J Dermatol 2010;20:489-496.

4 Pinter A, Pätzold S, Kaufmann R: Lichen planus of nails - successful treatment with alitretinoin. J Dtsch Dermatol Ges 2011;9:10331034.

5 Kolios AG, Marques Maggio E, Gubler C, et al: Oral, esophageal and cutaneous lichen ruber planus controlled with alitretinoin: case report and review of the literature. Dermatology 2013;226:302-310.

$\checkmark 6$ Le Cleach L, Chosidow O: Clinical practice. Lichen planus. N Engl J Med 2012;366:723-732.
7 Tosti A, Piraccini BM, Cambiaghi S, Jorizzo M: Nail lichen planus in children: clinical features, response to treatment, and long-term follow-up. Arch Dermatol 2001;137:10271032.

$>$ Ujille H, Shibaki A, Akiyama M, Shimizu H: Successful treatment of nail lichen planus with topical tacrolimus. Acta Derm Venereol 2010;90:218-219.

$\checkmark$ Irla N, Schneiter T, Haneke E, Yawalkar N: Nail lichen planus: successful treatment with etanercept. Case Rep Dermatol 2010;2:173-176. 
10 Utsu M, Hida T, Takahashi H, Yamashita T: Etanercept-induced lichen planus-like eruptions following the lines of Blaschko. Eur J Dermatol 2012;22:544-545.

-11 Manousaridis I, Manousaridis K, Peitsch WK, Schneider SW: Individualizing treatment and choice of medication in lichen planus: a step by step approach. J Dtsch Dermatol Ges 2013, Epub ahead of print.
12 Kato N, Ueno H: Isolated lichen planus of the nails treated with etretinate. J Dermatol 1993 20:577-580.

13 Mostafa WZ: Lichen planus of the nail: treatment with antimalarials. J Am Acad Dermatol 1989;20:289-290.

14 Alsenaid A, Lang A, Ruzicka T, Braun-Falco M, Wolf R: Lichen planus with associated myasthenia gravis-successful treatment with acitretin. Eur J Dermatol 2013;23:909-910.
5 Lo Muzio L, Santarelli A, Campisi G, Lacaita M, Favia G: Possible link between Hashimoto's thyroiditis and oral lichen planus: a novel association found. Clin Oral Investig 2013;17: 333-336.

16 Molin S, Ruzicka T: Oral alitretinoin in lichen planus: two case reports. Acta Derm Venereol 2010;90:523-524. 